\title{
Effects of Diaphragmatic Control on Multiparametric Analysis of the Nasal Inspiratory Pressure Test and Inspiratory Muscle Activity in Healthy Subjects
}

\section{Kadja Benício}

Universidade Federal do Rio Grande do Norte

Vanessa R. Resqueti

Universidade Federal do Rio Grande do Norte

Fernando A. L. Dias

Universidade Federal do Paraná

Francesca Pennati

Politecnico di Milano

Andrea Aliverti

Politecnico di Milano

Jéssica Medeiros

Universidade Federal do Rio Grande do Norte

Guilherme Augusto de Freitas Fregonezi ( $\nabla$ fregonezi.guilherme@gmail.com )

Universidade Federal do Rio Grande do Norte https://orcid.org/0000-0003-4938-7018

Research

Keywords: respiratory muscles, nasal inspiratory pressure, physiotherapy

Posted Date: June 12th, 2020

DOI: https://doi.org/10.21203/rs.3.rs-34266/v1

License: (c) (i) This work is licensed under a Creative Commons Attribution 4.0 International License.

Read Full License 


\section{Abstract}

Background:We investigated the influence of diaphragmatic activation control (diaphC) on the relaxation rate and contractile properties, as well as the electrical activity of inspiratory muscles, assessed noninvasively using the sniff inspiratory pressure test (SNIP) and surface electromyography, respectively, in healthy subjects.

Methods:Twenty-two subjects (10 men and 12 women) performed 10 sniff maneuvers in two different days: with and without diaphC instructions. For the SNIP test with diaphC, the subjects were instructed to perform intense activation of the diaphragm. The tests with the best SNIP values were used for analysis.

Results: The maneuver with diaphC when compared to the maneuver without diaphC exhibited significant lower values for: SNIP $(p<0.01)$, maximum relaxation rate (MRR) $(p<0.01)$, maximum rate of pressure development (MRPD) $(p<0.01)$ and electrical activity of the sternocleidomastoid $(S C M)(p<0.01)$, scalene $(\mathrm{SCL})(p=0.01)$ and intercostal $(\mathrm{Cl})(\mathrm{p}=0.03)$ muscles; as well as significant longer contraction (CT) times $(p=0.02)$. In addition, the decay constant $(\operatorname{tau}, \tau)$ and relaxation time $(1 / 2 R T)$ did not present any changes.

Conclusion:The diaphragmatic control performed during the SNIP test influences the inspiratory pressure and the contractile properties of inspiratory muscles. This occurs due to changes in the pattern of muscle recruitment, which change forcevelocity characteristics of the test. Thus, instruction on diaphC should be encouraged for better performance of the SNIP test and for evaluation targeting the diaphragm muscle activity.

\section{Background}

The measurement of respiratory muscle strength has been used as an index for early detection of pulmonary dysfunction, as well as a parameter to assess the progression of diseases that cause respiratory muscle strength impairment. Additionally, it provides prognostic and predictive information on patient survival [1, 2]. In clinical practice, the methods commonly used for respiratory muscle assessment measure the Maximum Expiratory Pressure (MEP) and Maximum Inspiratory Pressure (MIP), the latter being complemented by the Sniff Nasal Inspiratory Pressure (SNIP). The SNIP is a non-invasive maneuver in which the patient performs an inspiratory effort, via one nostril, quickly and strongly. The sniff is a maneuver considered more physiological and easier to understand and execute, especially for children and people with neuromuscular disorders [3, 4].

The diaphragm is the largest and the main muscle involved in breathing. Over the years, transdiaphragmatic pressure (PDI) has been described as the most accurate measure to assess the strength of the diaphragm muscle [5]. This variable was studied by Miller et al. [6] who compared the PDIs obtained by MIP to the ones obtained by sniff. The results showed that PDI derived from sniff was greater than from MIP, and the authors suggested this resulted because of the diaphragm muscle being 
activated in greater intensity during the sniff maneuver. Thus, the sniff maneuver was considered as a tool to detect diaphragmatic muscle weakness $[6,7]$.

The study of the SNIP test kinetics is suggested by the literature as a predictor inspiratory muscles of fatigue. This is represented by the relaxation rates described as maximum relaxation rate (MRR), halfrelaxation time $(1 / 2 \mathrm{RT})$ and constant curve of decay $(\tau$, tau) after a maximum voluntary contraction, in addition to the contractile properties of the diaphragm such as maximum rate of pressure development (MRPD) and contraction time (CT) [8].

Ventilation is a complex process involving several structures and systems. The literature is controversial on the evaluation of respiratory muscles, especially regarding the most common tests used in a clinical environment. Occasionally the shortage of details to describe clinical tests, such as the SNIP test, can lead to methodological errors and misinterpretation of results. The study carried out by Benício et al. [9] is, as far as we know, the only one that proposed to evaluate the influence of diaphragmatic control (diaphC) during the SNIP test. It reports that diaphC maneuvers generate lower SNIP values, possibly due to a lower recruitment of accessory respiratory muscles, in addition to generating tests with technical standardization. That being so, the characteristics of sniff maneuvers without diaphC are different, which determines errors in the test values. Therefore, it is necessary to investigate the importance of encouraging diaphragmatic contraction during the SNIP test in order to clarify the technical procedures for performing the maneuver.

The present study aims to evaluate the influence of diaphragmatic control on sniff nasal inspiratory pressure (1), relaxation rates and contractile properties of inspiratory muscles (2) and pattern of respiratory muscle activity (3) in healthy subjects.

\section{Methods}

\section{Type of study and subjects}

This cross-sectional, quasi-experimental study was carried out at the PneumoCardioVascular Lab at the Universidade Federal do Rio Grande do Norte / Empresa Brasileira de Serviços Hospitalares (UFRN / EBSERH). Twenty-two self-reported healthy subjects, of both genders, aged between 18 and 30 years were investigated. The inclusion criteria adopted were: body mass index (BMI) classified between 20$29.9 \mathrm{~kg} / \mathrm{m}^{2}$, absence of history of cardiovascular, neurological, pulmonary diseases or diagnosis of deviated septum, and presenting the spirometric variables of forced vital capacity (FVC) and forced expiratory volume ratio in the first second by the forced vital capacity $\left(\mathrm{FEV}_{1} / \mathrm{FVC}\right)$ greater than $80 \%$ and $85 \%$ of the predicted value [10], respectively. We excluded subjects that failed to perform the tests/protocol, as well as those who presented irregularities during data analysis or voluntarily requested their removal from the study. The project was approved by the Research Ethics Committee of HUOL (opinion 1.252.028 / 2015). All participants signed a consent form drafted in accordance with the Declaration of Helsinki. 


\section{Study design}

The subjects were initially submitted to anamnesis, clinical, spirometric and respiratory muscle strength assessment and after this initial screening the SNIP evaluation protocol was performed in two different days with an interval of 48 hours, in order to minimize the learning effect. Assessments on both days were conducted by the same evaluator and the maneuvers were carefully demonstrated prior to commencement of the test, subsequently the subject was asked to repeat the maneuver for the purpose of familiarization $[11,12]$. The SNIP assessments were carried out concomitantly with measurement of respiratory muscle activation using surface electromyography (sEMG). On day 1, sniff maneuvers without diaphragmatic control (without diaphC) were evaluated, the subjects were given the explanations following the guidelines of the American Thoracic Society / European Respiratory Society (ATS / ERS) [13]. On day 2, the subjects were initially trained to breathe with a slow diaphragmatic breathing pattern, being instructed to inhale deeply through the nose, while moving the abdominal wall outward. A period of 5 to 10 minutes of training was established so that patients could adapt to the pattern. Success was assessed visually, and the execution of the pattern was considered satisfactory when there was a clear visualization of the increase in abdominal volume during inspiration [14]. After training the diaphragmatic breathing pattern, the individual was asked to quickly inhale moving the abdomen outward in a ballistic manner to familiarize himself with the speed and strength to be performed during the sniff maneuvers. After these moments, he performed the sniff maneuvers associated to the abdominal movement (with diaphC). The same recommendations as day 1 , were given to perform the sniff with the addition of emphasis on diaphragmatic control during its execution.

\section{Lung function}

Spirometry was assessed using the KoKo DigiDoser® spirometer (Longmont, USA). The evaluations were carried out following the ATS / ERS [13] acceptability and reproducibility criteria and their reference values according to the values predicted for Brazilian adults [10]. Respiratory muscle strength was inferred by measuring the maximum inspiratory pressure (MIP), maximum expiratory pressure (MEP) and nasal inspiratory pressure (SNIP) using the digital manovacuometer (NEPEB-LabCare / UFMG, Belo Horizonte - MG, Brazil). The evaluations were carried out according to the ATS / ERS [13] acceptability and reproducibility criteria. For MIP and MEP, the reference values previously published by Neder et al. [15] were used, while for SNIP, the reference values of Araújo et al. [16] were used. For all pulmonary function variables, absolute values and the percentage of predicted values were used for analysis.

\section{Surface electromyography}

Surface electromyography was performed following the recommendations of the International Society of Electrophysiology and Kinesiology (ISEK) [17]. Myoelectric signals were recorded using the TeleMyo DTS Desk Receiver ${ }^{\circledR}$ device (Noraxon USA Inc., Scottsdale, USA) and 4 wireless sensors (Clinical DTS, Noraxon USA Inc., Scottsdale, USA) with a $20-500 \mathrm{~Hz}$ pass filter-band, 1000 gain, 16 -bit resolution, and a common mode rejection rate greater than $120 \mathrm{~dB}$. Bipolar double trace $\mathrm{Ag} / \mathrm{AgCl}$ (Miotec, Porto Alegre, Brazil) passive surface self-adhesive electrodes were placed on these following muscles: scalene (SCL), 
at a distance of 5 centimeters from the sternoclavicular joint and 2 centimeters above this point [18]; sternocleidomastoid (SCM), in the lower third of the distance between the mastoid process and the sternoclavicular joint [19]; and in the parasternal portion of the intercostal muscle (IC) over the second intercostal space and $3 \mathrm{~cm}$ from the sternum [20]. All electrodes were placed on the right side of the body to minimize cardiac noise interference. Before placing the electrodes, the skin region was trichotomized and cleaned with alcohol to reduce the impedance. The software used to capture, process and store the signals was the MR 3.2 (Noraxon. Inc., Scottsdale, USA). Raw data was analysed by means of RMS (root mean square) and standardized from respiratory baseline values [4].

\section{SNIP Curve Analysis}

The SNIP assessments (without diaphC and with diaphC) were performed from the Functional Residual Capacity (FRC). With the mouth closed, the subject performed a maximum sniff at the end of a slow and relaxed exhalation, responding to a verbal command of "pull". The evaluation was considered complete when 10 acceptable maneuvers were performed without air leakage and respecting a one-minute rest interval between them [21]. At the end of the measurement, the highest SNIP value obtained, which had a graph properly plotted, was used for data analysis. The criteria used to select the appropriate sniff maneuvers for analysis were: (1) peak pressure sustained for less than 50 milliseconds ( $\mathrm{ms}$ ) and without biphasic peak, (2) total sniff duration less than $500 \mathrm{~ms}$ and (3) shape of sniff pressure curve that presents a smooth decay [22].

The variables for the multiparametric analysis of the SNIP curve were calculated from the sniff maneuver trace. CT and $1 / 2$ RT were calculated as the time to reach the peak sniff pressure and the half-time of the relaxation curve, respectively [23]. MRPD, expressed in $\mathrm{cmH}_{2} \mathrm{O} \cdot \mathrm{ms}^{-1}$, was calculated as the negative peak

of the first derivative from the pressure-time curve [24], while MRR, expressed in milliseconds ${ }^{-1}$, was defined as the positive peak of the first derivative from the pressure-time curve normalized by the peak inhalation pressure, in order to make contractions of different intensities comparable [25]. The time constant ( $\tau$, tau) was calculated by plotting the natural logarithm of pressure as a function of time. The lower $50-70 \%$ of the pressure decay curve follows a straight line [25], indicating that the pressure follows a monoexponential decay with a time constant $\tau(\tau=1$ / slope). For the $\tau$ measure to be accepted, the correlation coefficient of the individual regression line (log(pressure) vs. time) should be $\geq 0.96$ [26]. SNIP curves were analyzed by custom software developed in MatLab (MathWorksInc, Natick, MA, USA) by TBM lab at Politecnico di Milano.

\section{Statistical analysis}

The sample size was established considering SNIP as the main variable. We analysed five subjects using a hypothetical paired $t$ test with mean and standard deviation (SD) for without DiaphC (96 \pm 16.7$)$ and with DiaphC $(76.8 \pm 22.8)$ groups. A sample of 14 subjects was estimated using an alpha error of 0.05 with bilateral distribution and a test power of $80 \%$. 
Data normality and distribution were verified using the Shapiro-Wilk test. The analyses between the different sniff maneuvers (Without DiaphC and With DiaphC) were studied using the paired t test or Wilcoxon for parametric and non-parametric data, respectively. For data analysis, the GraphPad Prism 6.0 program (GraphPad Software, San Diego, USA) was used. The study power ( $\beta$ ) and the effect size (ES) [27] were calculated and are detailed in the main study variables. The sample calculation, study power and effect size were analysed using the G*Power software, version 3.1.9.2 (Kiel, Germany). For all statistical analyses, a level of significance, $p<0.05$ with bilateral distribution was adopted.

\section{Results}

Thirty-four subjects were screened, of which 27 were included in the study. Five subjects were excluded after the complete evaluation due to low quality of their electromyographic signals and/or because it was not possible to obtain appropriate SNIP curves for graphic analysis, resulting in a final sample of 22 subjects (12 female, 10 male). The study design is shown in Fig. 1 . The sample anthropometric characteristics, pulmonary function and respiratory muscle strength data are shown in Table 1. 
Table 1

Sample description

\section{Description}

\begin{tabular}{|c|c|}
\hline Subjects (n) & 22 \\
\hline Gender F/M & $12 / 10$ \\
\hline Age, yrs & $23.7 \pm 2.2$ \\
\hline Weight (Kg) & $65.5 \pm 8.9$ \\
\hline Height (m) & $1.69 \pm 0.9$ \\
\hline BMI $\left(\mathrm{Kg} / \mathrm{m}^{2}\right)$ & $22.8 \pm 1.9$ \\
\hline \multicolumn{2}{|c|}{ Pulmonary function } \\
\hline $\mathrm{FEV}_{1} \mathrm{~L}$ & $3.79 \pm 0.73$ \\
\hline$\%$ predicted & $92.9 \pm 9.6$ \\
\hline FVC L & $4.49 \pm 0.94$ \\
\hline$\%$ predicted & $96.8 \pm 9.1$ \\
\hline $\mathrm{FEV}_{1} / \mathrm{FVC}$ & $0.85 \pm 0.06$ \\
\hline$\%$ predicted & $96.2 \pm 8.62$ \\
\hline MIP $\mathrm{cmH}_{2} \mathrm{O}$ & $121.5 \pm 31.6$ \\
\hline$\%$ predicted & $105.7 \pm 26.1$ \\
\hline MEP $\mathrm{cmH}_{2} \mathrm{O}$ & $118.7 \pm 28.5$ \\
\hline$\%$ predicted & $98.1 \pm 16.4$ \\
\hline
\end{tabular}

Values are mean \pm SD. F: female; M: male; yrs: years; BMI: Body Mass Index; kg: kilograms; m: meters; $\mathrm{FEV}_{1}$ : Forced Expiratory Volume in the first second; L: liters; FVC: Forced Vital Capability; MIP: Maximum Inspiratory Pressure; $\mathrm{CmH}_{2} \mathrm{O}$ : centimeters of water; MEP: Maximum Expiratory Pressure; SNIP: Sniff Nasal Inspiratory Pressure.

Figure 2 shows the parameters extracted from the multiparametric analysis of the SNIP curve. A significant reduction in SNIP, MRR and MRPD was observed in the maneuvers performed with DiaphC compared to without DiaphC $(p<0.01)$, however there was no statistical difference between MRR and MRPD after normalization ( $p=0.82$ and $p=0.31$ ). The variables related to relaxation, $\tau$ and $1 / 2 R T$, did not show differences between the maneuvers $(p=0.16$ and $p=0.13$, respectively). The CT was significantly higher in the DiaphC maneuver $(p=0.02)$. The total time $\left(T_{\text {TOT }}\right)$ did not vary between maneuvers $(p=$ 0.23). 
The RMS values for the respiratory muscles were normalized and are shown in Fig. 3. When performed the maneuver with DiaphC the \% RMS of the SCL, SCM and IC muscles $(p=0.01, p<0.01$ and $p=0.03$, respectively) showed lower values compared to the maneuver without DiaphC.

Figure 4 shows the represent of the SNIP test kinetics concomitant with the capture of electromyographic activity of SCM, SCL and IC muscles during maneuvers with and without diaphC, demonstrating the pressure reached, the total time of the maneuver, as well as the peak activity of the respiratory muscles assessed.

Table 2 reports the size effect size and power test for comparisons between maneuver with and without diaphC in the parameters obtained from the sniff nasal inspiratory pressure (SNIP) curve. MRR: maximum relaxation rate; MRPD: maximum rate of pressure development; CT: contraction time; $122 \mathrm{RT}$ : half-relaxation time; $\tau$ : tau and SEMG RMS values of SCM, SCL and IC muscles.

Table 2

Effect size and power test

\section{Comparisons between maneuver with and without DiaphC}

\begin{tabular}{|c|c|c|}
\hline & Effect size Cohen's dz & Power \\
\hline SNIP $\left(\mathrm{cmH}_{2} \mathrm{O}\right)$ & 1.68 & $<0.99$ \\
\hline $\operatorname{MRR}\left(\mathrm{cmH}_{2} \mathrm{O} / \mathrm{s}\right)$ & 0.97 & 0.99 \\
\hline MRR normalized & 0.27 & 0.23 \\
\hline MRPD $\left(\mathrm{cmH}_{2} \mathrm{O} / \mathrm{s}\right)$ & 1.06 & 0.99 \\
\hline MRPD normalized & 0.23 & 0.18 \\
\hline $1 / 2 \mathrm{RT}(\mathrm{s})$ & 0.32 & 0.3 \\
\hline $\mathrm{T}_{\text {ТОТ }}(\mathrm{s})$ & 0.26 & 0.21 \\
\hline CT (s) & 0.54 & 0.68 \\
\hline$\tau(s)$ & 0.14 & 0.09 \\
\hline SCM (\%RMS) & 0.64 & 0.71 \\
\hline SCL (\%RMS) & 0.59 & 0.63 \\
\hline IC (\%RMS) & 0.5 & 0.49 \\
\hline \multicolumn{3}{|c|}{$\begin{array}{l}\text { Comparisons between maneuver with and without DiaphC in the parameters obtained from the sniff } \\
\text { nasal inspiratory pressure (SNIP) curve and respiratory muscle activation. MRR: maximum relaxation } \\
\text { rate; MRPD: maximum rate of pressure development; } 1 / 2 \mathrm{RT} \text { : half-relaxation time; T } \mathrm{T}_{\mathrm{TOT}} \text { : total time; } \mathrm{CT} \text { : } \\
\text { contraction time; } \mathrm{t} \text { : tau; } \mathrm{cmH}_{2} \mathrm{O} \text { : centimeters of water; s: seconds; } \mathrm{SCM} \text { : sternocleidomastoid; } \mathrm{SCL} \text { : } \\
\text { scalene; IC: Intercostal; \%RMS: percentage of room mean square. }\end{array}$} \\
\hline
\end{tabular}




\section{Discussion}

The aim of this study was to evaluate the influence of diaphragmatic activation control on the variables resulting from the sniff nasal inspiratory pressure test and inspiratory muscle activity. Additionally, we aimed to determine the technical standardization for performing the sniff maneuver in healthy subjects.

The main findings of this study show that the maneuver with DiaphC compared to without DiaphC presents: 1) significantly lower SNIP value, 2) reduced activity of SCM, SCL and IC muscles; 3 ) reduced absolute values of MRR and MRPD, since they are directly proportional to the pressure obtained (dP / dt), however values normalized by the peak pressure showed no different behavior, and 4) increased contraction time.

Diaphragmatic activation control influences the SNIP, reducing its values. This finding supports the hypothesis that when performing ballistic contraction of the diaphragm muscle during a sniff maneuver, the diaphragm performs its action in a more isolated way which means the accessory muscles are less activated and, as a consequence, the SNIP values are lower. This is based on the findings described by Benício et al. [9].

During a breathing cycle, several muscles act on the rib cage to facilitate breathing. The contraction of diaphragm is directly related to the inspiration phase, while its relaxation favors the basal expiration. The expiration occurs passively as a result of the lungs elastic recoil. During the measurement of maximum respiratory pressures, the subject performs a forced inspiration, in which the diaphragm and respiratory accessory muscles act together.

The assessment of maximum respiratory pressures represents the measurement of respiratory muscle strength and can be assessed by volitional or non-volitional tests. Volitional tests are simple, portable and inexpensive; however they depende on maximum voluntary neuromuscular activation [28] which can be considered a limitation. MIP is the volitional test commonly used for inspiratory muscle strength assessment and it is measured during the performance of a maximum forced inspiration against a pressure gauge using a mouthpiece. It is sometimes considered difficult to perform, which may result in lower values when there are air leaks, as well as in cases of lack of motivation or coordination by the subject being assessed [29]. Currently, the SNIP test has been used to complement the MIP on the assessment of inspiratory muscles. For such, the physiologic sniff maneuver is performed with the occlusion of one nostril against a pressure manometer. As a more natural maneuver, the sniff is easy to perform [29], however, there is paucity of data regarding standardization of the SNIP test and the assessment of muscle recruitment patterns during the test.

Verin et al. [30] studied how voluntarily changing muscle recruitment affects sniff esophageal $\left(P_{E S}\right)$, gastric $\left(P_{G A}\right)$ and transdiaphragmatic $\left(P_{D I}\right)$ pressures. They assessed 3 different types of sniff maneuvers: natural, diaphragmatic and extra diaphragmatic. The results showed that in the natural sniff maneuver the subjects vary their recruitment pattern between diaphragmatic and extra diaphragmatic and that the performance of the diaphragmatic sniff maneuver presented higher values of 
transdiaphragmatic and gastric pressures compared to the other patterns, in addition to emphasizing in the conclusion of their results the need for studies that assess the benefits of abdominal dislocation (i.e. diaphragmatic control) during the SNIP test. Similar to our results, the study by Benício et al. [9] exposed that performing diaphragmatic control during the sniff maneuver results in lower SNIP value when compared to the maneuver without DiaphC and associated this to the probable difference in muscle recruitment during both maneuvers. These studies are, as far as we know, the only ones that proposed to evaluate the influence of diaphragmatic control during the SNIP test and our results complement the authors' interpretation.

The surface electromyography of the respiratory muscles evaluated during the SNIP test helps to clarify the results of this study. A reduction in the activity of respiratory accessory muscles was observed after training of diaphragmatic activation control (i.e. maneuver with DiaphC) when compared to without DiaphC. This indicates that despite the increase in transdiaphragmatic pressure in maneuvers with DiaphC [30], reducing the action of accessory muscles reduces SNIP values.

Previous studies have assessed the electromyographic activity of respiratory muscles during sniff maneuvers [31, 32]. Nava et al. [32] evaluated 3 different maximal inspiratory maneuvers, which demonstrated that the sniff maneuver has a higher diaphragmatic activation pattern, represented by higher values of diaphragmatic pressure and electrical activity of the diaphragm muscle. Additionally, they reported that the recruitment pattern of the inspiratory muscles of the rib cage were similar during sniff and Müller maneuvers. Katagiri et al. [31] evaluated the activation of accessory muscles during the sniff maneuver and demonstrated the performance of the scalene muscle during low intensity sniff and additional activity of the sternocleidomastoid muscle in high intensity sniff.

Therefore, we can infer that maneuvers with DiaphC present reduced SNIP values due to a decreased activity of the rib cage muscles, resulting in a more targeted expression of the diaphragm activity. The maneuvers without diaphC, on the other hand, recruit the respiratory accessory muscles more strongly and, therefore, the characteristics of the sniff maneuver are modified, which underestimates the test values.

The study of the SNIP test kinetics has been used as an indirect marker of muscle fatigue and inspiratory muscle overload and it is represented by the maximum relaxation rate (MRR). This occurred after Kyroussis et al. [33] reported that the MRR obtained non-invasively by performing a sniff maneuver reflects the value of the MRR measured at esophageal pressure curves.

Esau et al. $[7,34]$ suggested that the rate of decline in the PDI reflects the MRR, as the electrical activity of the diaphragm muscle ceases when the PDI decreases, therefore the beginning of the pressure drop coincides with the beginning of the diaphragm relaxation. MRR has been evaluated through analysis of the pressure curves of $\mathrm{P}_{\mathrm{DI}}[26,35], \mathrm{P}_{\mathrm{ES}}[36], \mathrm{P}_{\mathrm{ORAL}}[22,37]$ and SNIP in several studies. All of these studies assumed the variation of pressure reflects the changes in the diaphragm length-tension due to the coincidence between the interruption of diaphragm activity and the beginning of pressure decay. Several 
studies have shown that the slowdown in MRR indicates respiratory muscle fatigue, especially the diaphragm $[22,38]$.

Diaphragmatic control influenced the MRR reducing its values, however, when the MRR was normalized divided by the peak pressure obtained in the same maneuver, no difference was observed between sniff maneuvers with or without DiaphC. The interpretation of MRR values in this scenario should be cautious, considering that its reduced values in DiaphC maneuvers do not reflect muscle weakness, but rather a change in muscle recruitment - emphasising the diaphragm more than other inspiratory muscles. This change promoted the reduction of SNIP and consequently MRR, as the latter is a measure directly proportional to the inspiratory pressure obtained. The normalization of MRR by peak pressure (MRRnorm $=(\mathrm{dP} / \mathrm{dt}) / \mathrm{P}_{\mathrm{SNIP}}$ * 100) aims to exclude the effect of pressure oscillation amplitude [33], being the real representation of the relaxation rate.

Literature shows $\tau$ and $1 / 2$ RT as complementary in the evaluation of relaxation kinetics. These variables also did not show variations in the sniff maneuvers performed with and without DiaphC. This result was expected, since the experiment proposed to evaluate the influence of diaphragmatic control on maneuvers and not respiratory muscle fatigue. Thus, we can understand that the execution of diaphragmatic control did not change the relaxation kinetics in the evaluated subjects.

The contractile properties of respiratory muscles (MRPD and CT) are still poorly studied, being scarce in the literature. The MRPD represents the positive peak of the pressure derivative over time during the initial slope of the maximum respiratory pressure curve. Similar to the MRR, the MRPD it is under the effect of variations in pressure amplitude and due to this fact, it also had its values normalized by peak pressure. Tzelepis, Kasas and McCool [39] observed that muscle training protocols increased lung function by analysing the MRPD and showing that the increase in MPRD was directly proportional to the inspiratory pressure. Our results also followed this pattern, as such the MRPD values were higher in maneuvers without DiaphC. However, when normalized, the MRPD did not differ, showing that muscle activation in both maneuvers are equivalent.

Muscle fatigue is observed by an increase in contraction time. This is explained by a greater number of motor units recruited in situations of muscle stress. Our results show that DiaphC maneuvers showed lower CT values when compared to sniffs without DiaphC. Returning to the muscular arrangement characteristic of each maneuver, we explain this result, relating the recruitment primarily of only one muscle, the diaphragm, when performing the diaphragmatic control, ends up reducing the number of motor units and consequently the CT.

Benício et al. [9] also question the technical characteristics of sniff maneuvers. The authors reported $40 \%$ of the maneuvers without DiaphC exceeded the maneuver time recommended in the literature. This difference was not evidenced in the present study, since we adopted in our inclusion criteria the technical characteristics recommended in the literature [22] and therefore, none of the tests selected for evaluation 
presented a contraction time greater than $500 \mathrm{~ms}$. Additionally, no differences were found in the total time between the maneuvers.

In summary, our results show that diaphragmatic activation control modifies the kinetics of the SNIP test, emphasizing the action of the diaphragm muscle reducing the action of rib cage muscles, without changing the relaxation properties and improving the precision of the test to study diaphragm function. Thus, we suggest that interpretation of SNIP values depends on the purpose of the assessment. SNIP should be used as an indicator of global inspiratory muscle function when the maneuver without DiaphC is adopted, emphasizing that inspiratory pressure values resulting from this maneuver underestimate the SNIP. Alternatively, maneuvers with DiaphC should be prioritized in assessing muscle function directly related to the diaphragm.

The study showed limitations in its evaluation format. The direct evaluation of the diaphragm muscle electrical activity would reinforce our results. However, we were unable to capture the activity of this muscle using SEMG and we could not use invasive resources for this measurement. Nevertheless, it is important to note that same conditions were applied in both assessments to allow for comparison. In addition, we emphasize that applying this technique can direct the results of the maneuver and assist with interpretation of its values.

\section{Conclusions}

Encouraging diaphragmatic contraction changes the characteristics of the SNIP test. The mane uvers without DiaphC recruit respiratory accessory muscles more strongly and, therefore, the characteristics of the sniff maneuver are modified, which determines higher test values.

We conclude that for a more effective assessment of diaphragmatic strength, the diaphragmatic control should be encouraged during the SNIP test. The maneuver must be explained in detail and demonstrated by the examiner, since it allows for a more accurate measurement within the standards described in literature.

\section{Abbreviations}

$1 / 2$ RT

half-relaxation time; ATS/ERS:American Thoracic Society / European Respiratory Society; BMI

Body Mass Index; $\mathrm{cmH}_{2} \mathrm{O}$ :centimeters of water; $\mathrm{CT}$ :contraction times; diaphC:diaphragmatic control; EBSERH:Empresa Brasileira de Serviços Hospitalares; FEV 1 :Forced Expiratory Volume in the first second; FRC:Functional Residual Capacity; FVC:Forced Vital Capability; IC:intercostal; ISEK:International Society of Electrophysiology and Kinesiology; kg:kilograms; L:liters; m:meters; MEP:Maximum Expiratory Pressure; MIP:Maximum Inspiratory Pressure; MRPD:maximum rate of pressure development; MRR:maximum relaxation rate; $m s$ :milliseconds; $\mathrm{P}_{\mathrm{DI}}$ :transdiaphragmatic pressure; $\mathrm{P}_{\mathrm{ES}}$ :esophageal 
pressure; $\mathrm{P}_{\mathrm{GA}}$ :gastric pressure; $\mathrm{P}_{\mathrm{ORAL}}$ :oral pressure; RMS:root mean square; SCL:scalene; SCM:sternocleidomastoid; sEMG:surface electromyography; SNIP:sniff inspiratory pressure test; UFRN:Universidade Federal do Rio Grande do Norte;t:tau.

\section{Declarations}

\section{Ethics approval and consent to participate}

The project was approved by the Research Ethics Committee of HUOL (opinion 1.252.028 / 2015). All participants signed a consent form drafted in accordance with the Declaration of Helsinki.

\section{Consent for publication}

Not applicable

\section{Availability of data and materials}

The datasets used and/or analysed during the current study are available from the corresponding author on reasonable request.

\section{Competing interests}

Not applicable

\section{Funding}

The study received financial support from the Coordenação de Aperfeiçoamento de Pessoal de Nível Superior (CAPES) - Finance Code 001 and PGCI CAPES 054/2014-23038.007514/2014-78. Guilherme Augusto de Freitas Fregonezi received a grant from Conselho Nacional de Desenvolvimento Científico e Tecnológico (CNPq) number 312876/2018-1, and Vanessa Resqueti received a grant from CNPq number 315580/2018-6.

\section{Authors' contributions}

The authors take responsibility for the integrity of the work as a whole, contributed to the writing and reviewing of the manuscript, and have given final approval for the version to be published. All authors had full access to the data in this study and take complete responsibility for the integrity of the data and accuracy of the data analysis. GF, AA and FD contributed to the conception or design of the study, and the data analysis and interpretation. JM, KB, VR, FD, FP, AA and GF contributed to the data analysis and interpretation. JM and $\mathrm{KB}$ contributed to the acquisition of data and the data analysis and interpretation. 


\section{Acknowledgements}

Not applicable

Authors' information

${ }^{1}$ PneumoCardioVascular Lab/HUOL, Hospital Universitário Onofre Lopes, Empresa Brasileira de Serviços Hospitalares and Departamento de Fisioterapia Universidade Federal do Rio Grande do Norte, Natal, Brazil

${ }^{2}$ Laboratório de Inovação Tecnológica em Reabilitação, Departamento de Fisioterapia, Universidade Federal do Rio Grande do Norte, Natal, Brazil

${ }^{3}$ Departamento de Fisiologia, Universidade Federal do Paraná (UFPR), Curitiba, PR, Brazil

${ }^{4}$ Dipartimento di Elettronica, Informazione e Bioingegneria, Politecnico di Milano, Milan, Italy

\section{References}

1. Morgan RK, McNally S, Alexander M, Conroy R, Hardiman O, Costello RW: Use of Sniff nasalinspiratory force to predict survival in amyotrophic lateral sclerosis. Am J Respir Crit Care Med 2005, 171:269-274.

2. Iandelli I, Gorini M, Misuri G, Gigliotti F, Rosi E, Duranti R, Scano G: Assessing inspiratory muscle strength in patients with neurologic and neuromuscular diseases: comparative evaluation of two noninvasive techniques. Chest 2001, 119:1108-1113.

3. Fauroux B, Aubertin G, Cohen E, Clément A, Lofaso F: Sniff nasal inspiratory pressure in children with muscular, chest wall or lung disease. European Respiratory Journal 2009, 33:113-117.

4. Stefanutti D, Benoist MR, Scheinmann P, Chaussain M, Fitting JW: Usefulness of sniff nasal pressure in patients with neuromuscular or skeletal disorders. Am J Respir Crit Care Med 2000, 162:15071511.

5. Davis J, Goldman M, Loh L, Casson M: Diaphragm function and alveolar hypoventilation. Q J Med $1976,45: 87-100$.

6. Miller JM, Moxham J, Green M: The maximal sniff in the assessment of diaphragm function in man. Clin Sci (Lond) 1985, 69:91-96.

7. Esau SA, Bye PT, Pardy RL: Changes in rate of relaxation of sniffs with diaphragmatic fatigue in humans. J Appl Physiol Respir Environ Exerc Physiol 1983, 55:731-735.

8. Sarmento A, Aliverti A, Marques L, Pennati F, Dourado-Júnior ME, Fregonezi G, Resqueti V: Multiparametric Analysis of Sniff Nasal Inspiratory Pressure Test in Middle Stage Amyotrophic Lateral Sclerosis. Frontiers in Neurology 2018, 9:306. 
9. Benício K, Dias FAL, Gualdi LP, Aliverti A, Resqueti VR, Fregonezi GAF: Effects of diaphragmatic control on the assessment of sniff nasal inspiratory pressure and maximum relaxation rate. Brazilian Journal of Physical Therapy 2016, 20:96-103.

10. Alberto de Castro Pereira C, Sato T, Carla Rodrigues S. Novos valores de referência para espirometria forçada em brasileiros adultos de raça branca. 2007.

11. Terzi N, Corne F, Mouadil A, Lofaso F, Normand H: Mouth and nasal inspiratory pressure: learning effect and reproducibility in healthy adults. Respiration 2010, 80:379-386.

12. Lomax M, McConnell AK: Influence of prior activity (warm-up) and inspiratory muscle training upon between- and within-day reliability of maximal inspiratory pressure measurement. Respiration 2009, 78:197-202.

13. Laveneziana $P$, Albuquerque A, Aliverti A, Babb T, Barreiro E, Dres M, Dubé B-P, Fauroux B, Gea J, Guenette JA, et al: ERS Statement on Respiratory Muscle Testing at Rest and during Exercise. European Respiratory Journal 2019:1801214.

14. Vieira DS, Mendes LP, Elmiro NS, Velloso M, Britto RR, Parreira VF: Breathing exercises: influence on breathing patterns and thoracoabdominal motion in healthy subjects. Braz J Phys Ther 2014, 18:544-552.

15. Neder JA, Andreoni S, Lerario MC, Nery LE: Reference values for lung function tests: II. Maximal respiratory pressures and voluntary ventilation. Brazilian Journal of Medical and Biological Research $1999,32: 719-727$.

16. Araújo PRS, Resqueti VR, Nascimento Jr J, Carvalho LdA, Cavalcanti AGL, Silva VC, Silva E, Moreno MA, Andrade AdFDd, Fregonezi GAdF: Valores de referência da pressão inspiratória nasal em indivíduos saudáveis no Brasil: estudo multicêntrico. Jornal Brasileiro de Pneumologia 2012, 38:700-707.

17. Merletti R, Farina D, Granata A: Non-invasive assessment of motor unit properties with linear electrode arrays. Electroencephalogr Clin Neurophysiol Supp/ 1999, 50:293-300.

18. Cunha APNM, P. E. M.; Silva, T. N. S.; França, E. E. T; Amorin, C.; Galindo Filho, V. C.; Andrade, A. D.: Efeito do Alongamento sobre a Atividade dos Musculos Inspiratórios na DPOC. Saúde Rev 2005, 7:13-19.

19. Falla D, Dall'Alba P, Rainoldi A, Merletti R, Jull G: Location of innervation zones of sternocleidomastoid and scalene muscles-a basis for clinical and research electromyography applications. Clin Neurophysiol 2002, 113:57-63.

20. Maarsingh EJ, van Eykern LA, Sprikkelman AB, Hoekstra MO, van Aalderen WM: Respiratory muscle activity measured with a noninvasive EMG technique: technical aspects and reproducibility. J Appl Physiol (1985) 2000, 88:1955-1961.

21. Heritier F, Rahm F, Pasche P, Fitting JW: Sniff nasal inspiratory pressure. A noninvasive assessment of inspiratory muscle strength. Am J Respir Crit Care Med 1994, 150:1678-1683.

22. Koulouris N, Vianna LG, Mulvey DA, Green M, Moxham J: Maximal relaxation rates of esophageal, nose, and mouth pressures during a sniff reflect inspiratory muscle fatigue. Am Rev Respir Dis 1989, 
139:1213-1217.

23. Bellemare F, Bigland-Ritchie B, Woods JJ: Contractile properties of the human diaphragm in vivo. $J$ Appl Physiol (1985) 1986, 61:1153-1161.

24. Romer LM, McConnell AK: Specificity and reversibility of inspiratory muscle training. Med Sci Sports Exerc 2003, 35:237-244.

25. Mador MJ, Kufel TJ: Effect of inspiratory muscle fatigue on inspiratory muscle relaxation rates in healthy subjects. Chest 1992, 102:1767-1773.

26. Wilcox PG, Eisen A, Wiggs BJ, Pardy RL: Diaphragmatic relaxation rate after voluntary contractions and uni- and bilateral phrenic stimulation. J Appl Physiol (1985) 1988, 65:675-682.

27. Lakens $D$ : Calculating and reporting effect sizes to facilitate cumulative science: a practical primer for t-tests and ANOVAs. Front Psychol 2013, 4:863.

28. Fitting J-W: Sniff nasal inspiratory pressure: simple or too simple? European Respiratory Journal $2006,27: 881-883$.

29. Lofaso F, Nicot F, Lejaille M, Falaize L, Louis A, Clement A, Raphael J-C, Orlikowski D, Fauroux B: Sniff nasal inspiratory pressure: what is the optimal number of sniffs? European Respiratory Journal 2006, 27:980-982.

30. Verin E, Delafosse C, Straus C, Morélot-Panzini C, Avdeev S, Derenne J-P, Similowski T: Effects of muscle group recruitment on sniff transdiaphragmatic pressure and its components. European Journal of Applied Physiology 2001, 85:593-598.

31. Katagiri M, Abe T, Yokoba M, Dobashi Y, Tomita T, Easton PA: Neck and abdominal muscle activity during a sniff. Respir Med 2003, 97:1027-1035.

32. Nava S, Ambrosino N, Crotti P, Fracchia C, Rampulla C: Recruitment of some respiratory muscles during three maximal inspiratory manoeuvres. Thorax 1993, 48:702-707.

33. Kyroussis D, Mills G, Hamnegard CH, Wragg S, Road J, Green M, Moxham J: Inspiratory muscle relaxation rate assessed from sniff nasal pressure. Thorax 1994, 49:1127-1133.

34. Esau SA, Bellemare F, Grassino A, Permutt S, Roussos C, Pardy RL: Changes in relaxation rate with diaphragmatic fatigue in humans. J Appl Physiol Respir Environ Exerc Physiol 1983, 54:1353-1360.

35. Aubier M, Murciano D, Lecocguic Y, Viires N, Pariente R: Bilateral phrenic stimulation: a simple technique to assess diaphragmatic fatigue in humans. J Appl Physiol (1985) 1985, 58:58-64.

36. Kyroussis D, Polkey MI, Keilty SE, Mills GH, Hamnegard CH, Moxham J, Green M: Exhaustive exercise slows inspiratory muscle relaxation rate in chronic obstructive pulmonary disease. Am J Respir Crit Care Med 1996, 153:787-793.

37. Levy RD, Esau SA, Bye PT, Pardy RL: Relaxation rate of mouth pressure with sniffs at rest and with inspiratory muscle fatigue. Am Rev Respir Dis 1984, 130:38-41.

38. Mulvey DA, Koulouris NG, Elliott MW, Laroche CM, Moxham J, Green M: Inspiratory muscle relaxation rate after voluntary maximal isocapnic ventilation in humans. J Appl Physiol (1985) 1991, 70:21732180. 
39. Tzelepis GE, Kasas V, McCool FD: Inspiratory muscle adaptations following pressure or flow training in humans. Eur J Appl Physiol Occup Physiol 1999, 79:467-471.

\section{Figures}

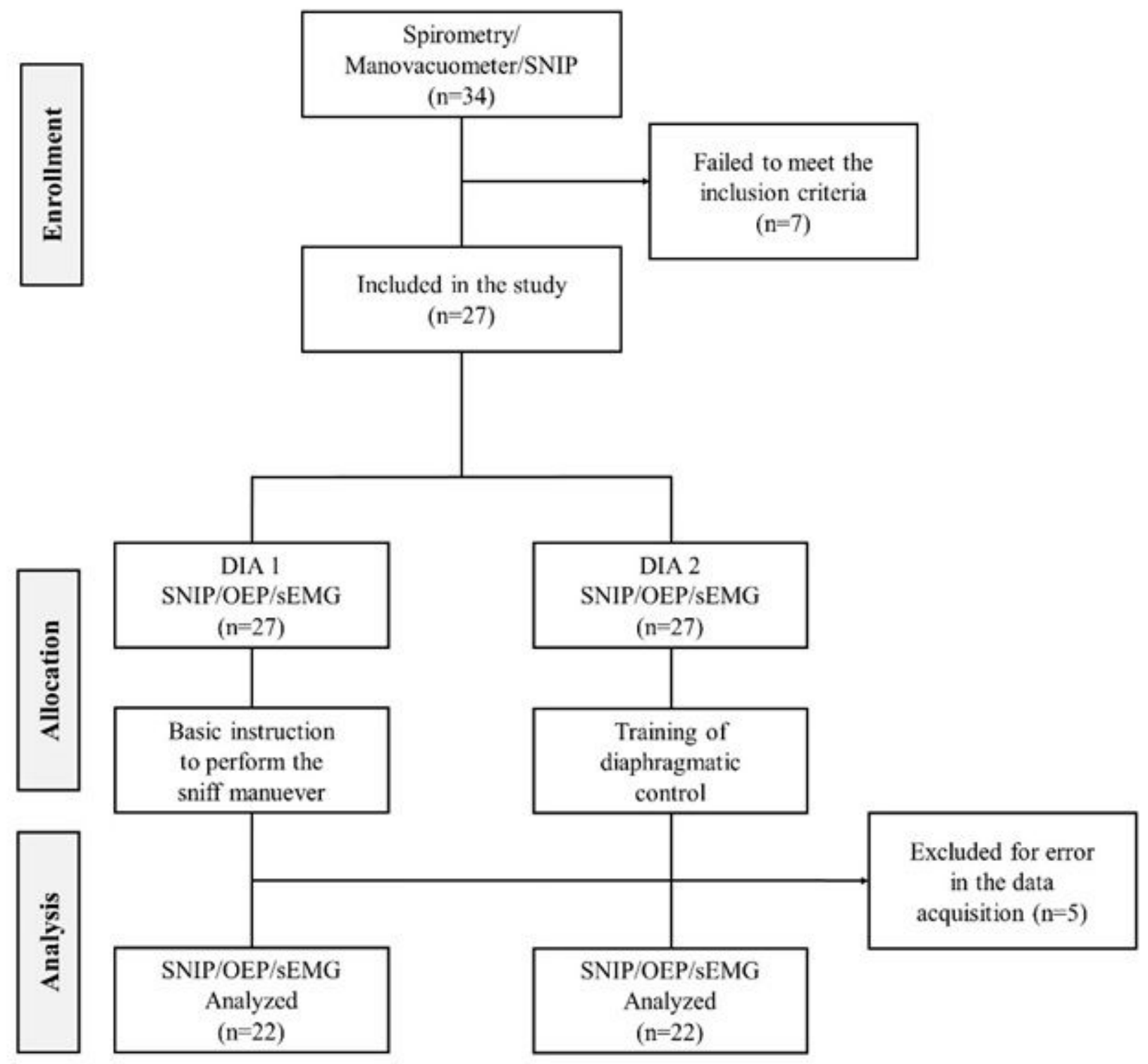

Figure 1

Study design 

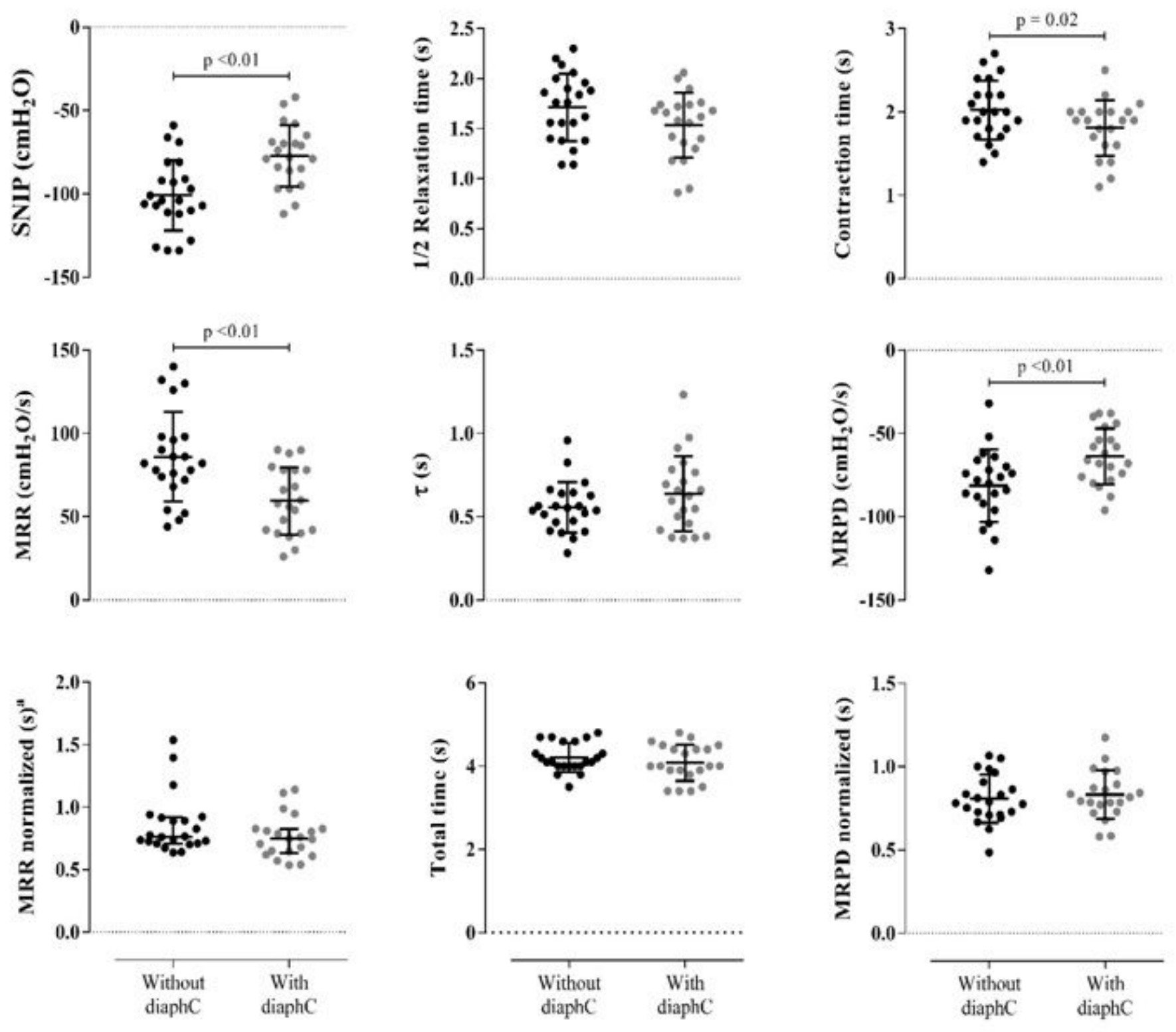

Figure 2

Data are shown as mean \pm SD. Comparisons between maneuver with and without diaphC in the parameters obtained from the sniff nasal inspiratory pressure (SNIP) curve. MRR: maximum relaxation rate; MRPD: maximum rate of pressure development; $\mathrm{CT}$ : contraction time; $1 / 2 \mathrm{RT}$ : half-relaxation time; $\tau$ : tau; $\mathrm{cmH} 2 \mathrm{O}$ : centimeters of water; s: seconds. 
SCM

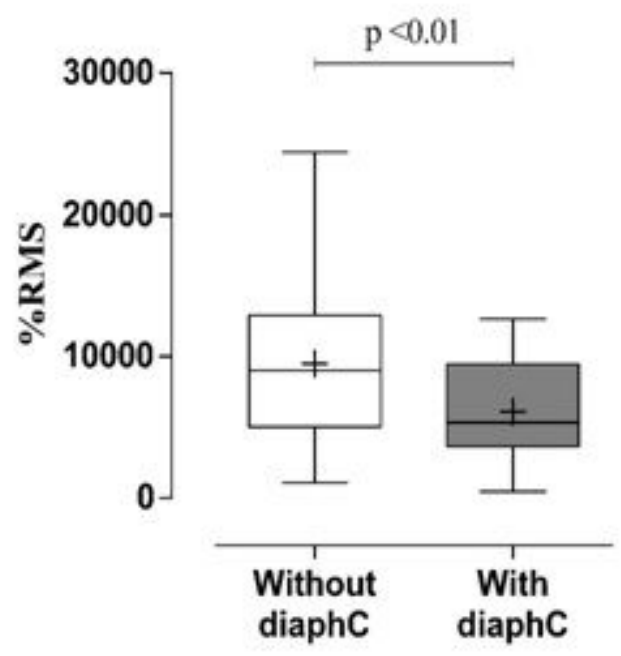

SCL

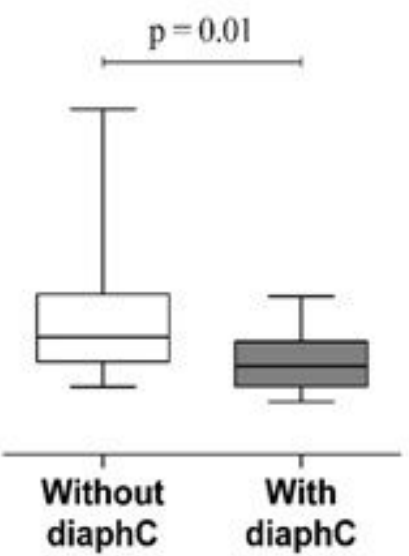

IC

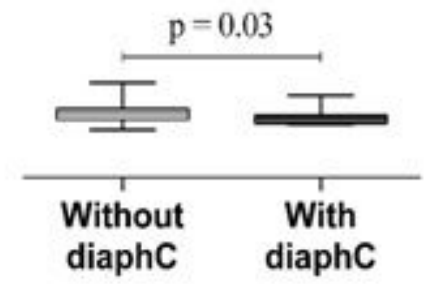

\section{Figure 3}

Data are shown as median [25-75th percentile]. Percentage of respiratory muscle activation during the maneuver with and without diaphC. SCM:sternocleidomastoid; SCL: scalene; IC: Intercostal; +, mean for parametric analysis. 

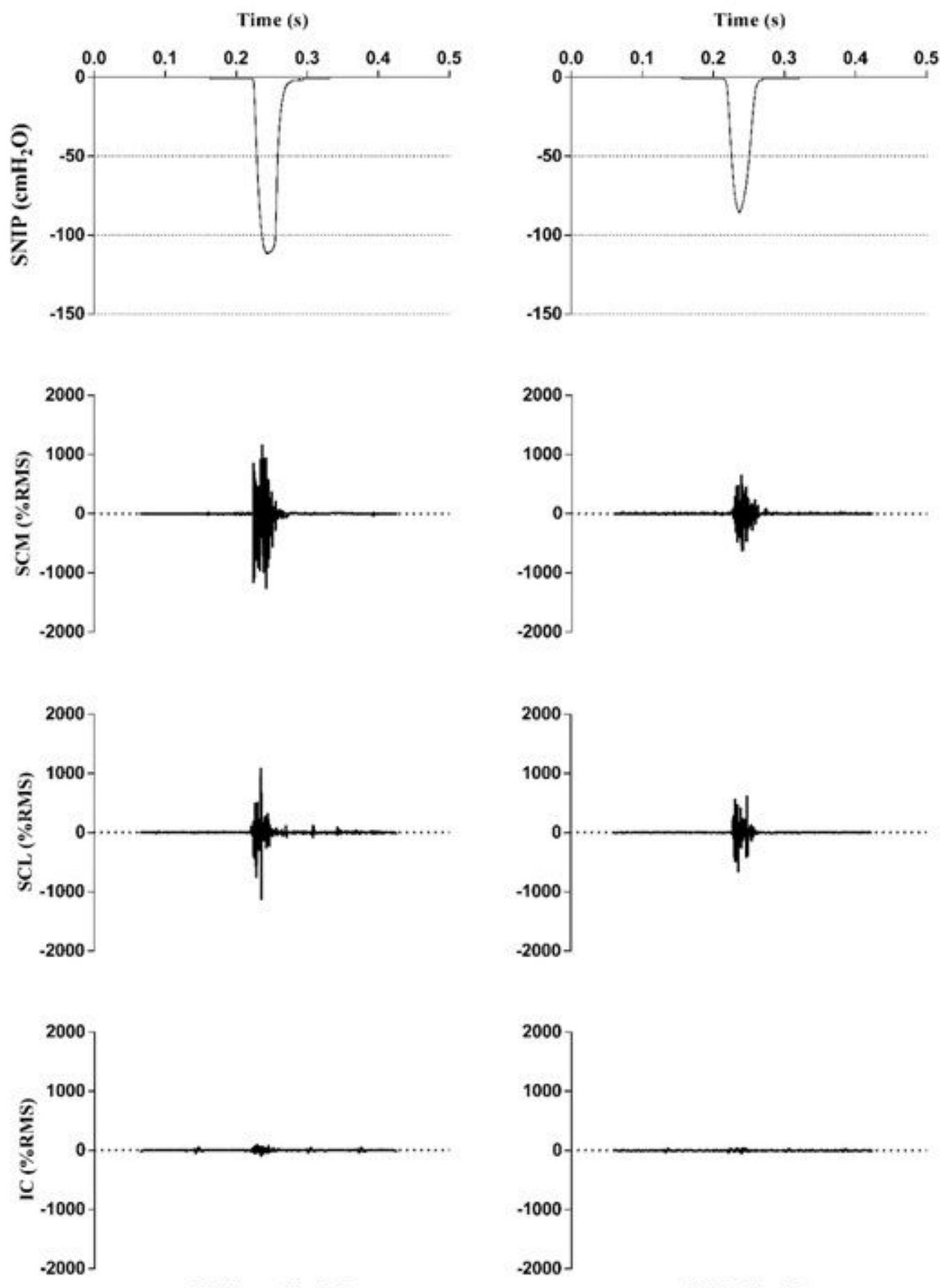

Without diaphC

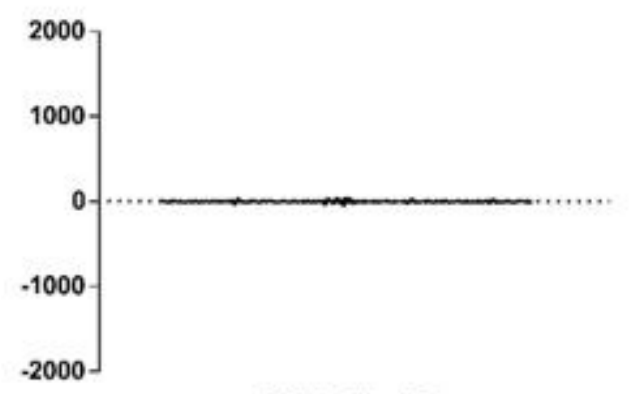

With diaphC

\section{Figure 4}

Scalar tracing of SNIP and electrical activity of scalene (SCL), sternocleidomastoid (SCM) and intercostal (IC) muscles, during the maneuver with and without diaphC. 